when the time for action comes. Even at the present moment we can no longer regard the Society merely as one to forward mathematical research in America; the wider interests of the subject have now to be considered, since we form the one large national organization devoted to research in mathematics which can continue its work unhindered by more pressing claims. In these wider interests our opportunities for service may come: the readiness and ability that we show in a realization and fulfilment of them will be the chief measure of our success in the past and promise for the future.

YALE UNIVERSITY, New Haven, Conn.

\title{
A THEOREM ON THE CURVES DESCRIBED BY A SPHERICAL PENDULUM.
}

\author{
BY PROFESSOR ARNOLD EMCH.
}

(Read before the American Mathematical Society, December 27, 1916.)

1. IN what follows I shall restrict myself to the case considered by Greenhill,* in which the pendulum bob reaches (but does not go above) the horizontal plane of suspension with a non-vanishing horizontal velocity. $\dagger$

When this assumption is made, there exists an interesting relation between the curve described by the bob and a certain quartic in space. It is the purpose of this note to establish this simple relation.

2 . Let $r_{0}$ be the distance of the bob from the vertical axis when its horizontal projection crosses the $x$-axis; $A+2 \pi$ the horizontal angle between two consecutive positions in the plane of suspension; $4 K$ the real period in the Jacobian elliptic functions appearing in the solution; $v=t / 2 w_{1}$, where $w_{1}$ is the real half-period in the Weierstrassian form, and $t$ the time; $a, b, c(a>b>c)$ the real roots of the cubic in the general solution; $\mu=\sqrt{\left(l^{2}+a^{2}\right) /\left(l^{2}-a^{2}\right)}$, where $l$ is the radius of

* Greenhill, Les Fonctions elliptiques et leurs Applications, chap. III. Appell, Traité de Mécanique rationnelle, t. 1, p. 494. Tannery et Molk, Eléments de la Théorie des Fonctions elliptiques, t. 4, p. 188.

$\uparrow$ The statement by L. Lévy, on page 161 of his Fonctions elliptiques, that in this case $x, y, z$ are doubly periodic functions of the time is not correct. 
the sphere. As in this case $b=0$, we find, according to Tannery, loc. cit., the positive sense of the z-axis being downward:

(1) $x=r_{0}[\cos (A+\pi) v \cdot \operatorname{cn}(2 K v)$

$$
-\mu \sin (A+\pi) v \cdot \operatorname{sn}(2 K v) \operatorname{dn}(2 K v)],
$$

(2) $y=r_{0}[\sin (A+\pi) v \cdot \operatorname{cn}(2 K v)$

(3) $z=a \mathrm{cn}^{2}(2 K v)$,

$$
+\mu \cos (A+\pi) v \cdot \operatorname{sn}(2 K v) \operatorname{dn}(2 K v)],
$$

for the coordinates of the bob in parametric form.

From (1) and (2) it is easily seen that

$$
\begin{aligned}
x \cos \phi+y \sin \phi & =r_{0} \operatorname{cn}(2 K v), \\
-x \sin \phi+y \cos \phi & =\mu r_{0} \operatorname{sn}(2 K v) \operatorname{dn}(2 K v),
\end{aligned}
$$

where $\phi=(A+\pi) v=\left(A+\pi / 2 w_{1}\right) t$ is an angle proportional to the time.

If we now put

$$
\begin{aligned}
& x^{\prime}=r_{0} \operatorname{cn}(2 K v), \\
& y^{\prime}=\mu r_{0} \operatorname{sn}(2 K v) \operatorname{dn}(2 K v), \\
& z^{\prime}=a \operatorname{cn}^{2}(2 K v),
\end{aligned}
$$

it is seen that the locus of the points $\left(x^{\prime}, y^{\prime}, z^{\prime}\right)$ is a quartic in space. For each of the functions $\operatorname{sn}(2 K v), \operatorname{cn}(2 K v), \operatorname{dn}(2 K v)$, has $i K^{\prime} / 2 K$ and $\left(i K^{\prime}+2 K\right) / 2 K$ as the only two poles within the period parallelogram, so that any linear combination

$$
\alpha x^{\prime}+\beta y^{\prime}+\gamma z^{\prime}+\delta
$$

is an elliptic function with four poles (counting multiplicities properly), and consequently four zeros within the period parallelogram. The curve is therefore cut in four points by every plane and is a quartic.

The coordinates of a point $P(x, y, z)$ of the pendulum curve and the corresponding point $P^{\prime}\left(x^{\prime}, y^{\prime}, z^{\prime}\right)$ of the quartic are connected by the formulas

$$
\begin{aligned}
& x^{\prime}=x \cos \phi+y \sin \phi, \\
& y^{\prime}=-x \sin \phi+y \cos \phi, \\
& z^{\prime}=z .
\end{aligned}
$$


Hence, as $x, y, z$ are functions of the time, the point $P^{\prime}$ is obtained by rotating the point $P$ (in the negative sense) about the vertical axis (z-axis), through an angle which is proportional to the time $t$, with $-\left[(A+\pi) / 2 w_{1}\right]$ as a proportionality factor. Hence the

THEOREM: If the points $P$ of the curve of a spherical pendulum motion, which just reaches the horizontal plane of suspension with a non-vanishing horizontal velocity component, are rotated in the negative sense about the vertical axis through angles proportional to the times (with a definite proportionality factor) associated with those points, the locus of the rotated positions of the P's is a quartic in space of the first kind.

4. The elimination of $v$ between (6) and (8) leads to the equation of the parabolic cylinder

$$
a x^{\prime 2}-r_{0}^{2} z^{\prime}=0 .
$$

It touches the plane of suspension along the $y$-axis, it is below this plane, and is symmetrical with respect to the $y z$-plane. The elimination of $v$ between (7) and (8) gives the equation

$$
a^{2}{y^{\prime}}^{2}+\mu^{2} r_{0}^{2} k^{2}(a-z)^{2}-\mu^{2} r_{0}^{2} a(a-z)=0,
$$

which represents an elliptical cylinder. It cuts the z-axis at a distance $a$ below and at a distance $a\left[\left(k^{2}-1\right) / k^{2}\right]$ above the origin. The quartic is now obtained as the intersection of these two cylinders and consists of only one real branch. The horizontal projection of this curve is a plane quartic of deficiency 1 , and with the equation

$$
\mu^{2} k^{2}{x^{\prime}}^{4}+\mu^{2} r_{0}^{2}\left(1-2 k^{2}\right) x^{\prime 2}+r_{0}{y^{\prime}}^{2}-\mu^{2} r_{0}^{4}\left(1-k^{2}\right)=0 .
$$

The infinite point of the $y$-axis is an isolated point which must be counted for two double points.

The converse of the theorem is evidently also true, i. e.,

There always exists a quartic of the first kind in space, such that the rotations of $i$ ts points about the vertical axis through certain definite angles proportional to the time, will lead to a given spherical pendulum curve, as specified above.

UNIVERSITY OF ILLINOIS. 